\title{
Conditionally-Sampled Estimates of Turbulent Scalar Flux in a Simulated Fire
}

\author{
N. L. CRAUFORD \\ Schlumberger Cambridge Research Ltd. \\ Cambridge, England
}

\author{
K. N. C. BRAY \\ University Engineering Department \\ Cambridge, England
}

J. B. Moss

Cranfield Institute of Technology

Bedford, England

\section{ABSTRACT}

A conditional sampling strategy is described in which temperature, measured by fine wire thermocouple, is used to trigger the acquisition of velocity data by IDA. The technique permits the determination of a range of scalar-velocity correlations, important to the development of flow field models, throughout a simulated buoyant fire. The experimental data is incorporated into flux estimates which permit the assessment of mass, energy and momentum balances in two distinctive, intermittently burning regimes in the buoyant flame.

\section{INTRODUCTION}

Natural fires are distinguished by the low initial momentum of the fuel source. Subsequent fuel-air mixing and combustion are then strongly influenced by buoyancy. The large-scale inhomogenities characteristic of buoyant mixing pose particular difficulties in respect of both mathematical models, seeking to predict rates of fire spread and flame shape, for example, and experimental techniques, providing detailed information to guide and evaluate such models. Several studies have been reported recently on turbulent flames subject to some degree of buoyant influence which attempt detailed diagnosis of flame structure and development $(1-6)$. Probe techniques, notably fine wire thermocouples and ionisation probes, and laser Doppler anemometry have been variously employed in determinations of time-mean and fluctuating components of velocity and temperature. Such measurements are not free from ambiguity since flame features of differing scales and light scattering particles appear to propagate at different mean speeds (6). However, despite this ambiguity, the microscopic insights provided by LDA do discriminate between important alternative modelling prescriptions. The present paper seeks to expand this experimental base by reporting direct measurements of the turbulent heat flux, $\overline{u^{\prime} T^{\prime}}$, and related flow properties in a turbulent buoyant flame. The joint determination of fluctuating velocity and temperature is effected by conditional sampling of LDA velocity using temperature, measured by a fine wire thermocouple, to trigger data acquisition.

Some recent comparisons between numerical prediction and experiment have been encouraging $(4,7)$. Two-equation $(k-\varepsilon)$ turbulence modelling, incorporating some buoyant influences and improved chemistry prescriptions, for example a conserved scalar formulation with flamelet chemistry, has permitted some macroscopic features, flame shape and mean properties, to be plausibly reproduced.

Considerable uncertainty however surrounds the most widely suitable 
representation of the large-scale, unsteady flame motion so characteristic of buoyant fires. Flame front flapping occurs, for example, at low frequency ( 3 $\mathrm{Hz}$ ), on scales comparable with the dimensions of the fire and may be highly anisotropic. The turbulent transport which results from such behaviour and which in many respects more closely resembles a buoyant instability than fullydeveloped eddy transport is then poorly represented. Even the sense of the turbulent flux, positive or negative, is uncertain in different parts of the intermittently-burning turbulent flame. Preliminary investigations into the simultaneous determination of velocity and temperature were reported by Walker and Moss (6) and we report here the results of more extensive mapping of a simulated fire together with an assessment of their implications for control volume balances of mass, momentum and energy. The particular interest in fire studies is often to integrate the source into more general descriptions of air and combustion products movement in enclosures and this has focussed attention on overall property balances. The contribution of turbulent fluxes to such balances are frequently neglected. Joint measurements in buoyant flames have to date been restricted to non-combusting plume regions, for example Nakagome and Hirata (8).

Walker and Moss (6) discuss the advantages in buoyant flame applications of LDA signal processing and analysis by photon correlation in comparison with alternative techniques which rely on substantial artificial seeding. Although the correlation technique permits velocity determination at the low signal-tonoise levels characteristic of laser light scattered from naturally occurring seed particles, the velocity-time series is then made inaccessible. Direct cross-correlation between velocity and temperature series is thus not possible and a conditional sampling strategy at the time of data acquisition must be employed.

\section{EXPERTMENTAL DETAIL}

The simulated fire comprises a porous refractory burner, $25 \mathrm{~cm}$ in diameter, fuelled by natural gas from the domestic supply. The exit fuel velocity is $0.017 \mathrm{~ms}^{-1}$, giving an approximate heat output of $28 \mathrm{~kW}$ and luminous flame height of roughly $0.9 \mathrm{~m}$. The burner is mounted on a traverse having two degrees of freedom, permitting movement of the flame in the vertical and horizontal directions. A minimum separation of $25 \mathrm{~cm}$ is maintained between burner face and floor to avoid air movement restrictions which might disturb the flame. The burner is located in a test area $3 \mathrm{~m}$ square by $4 \mathrm{~m}$ high, enclosed by solid walls on three sides and protected from draughts by a fine nylon mesh, screening the fourth side.

Complete details of the LDA system and method of data reduction are described by Crauford (9). Briefly, the digital photon correlator constructs the auto-correlation function of 1 ight scattered from naturally occurring soot particles as they traverse the LDA fringe field. This function is an ensemble average of many scattering events and joint velocity - temperature measurements can only be obtained by direct conditioning of LDA velocity acquisition. The latter is triggered by an externally generated strobe signal and the autocorrelation function is constructed in a piecewise manner from the selected events. A fine wire thermocouple, positioned Im upstream of the LDA fringe volume, provides a continuous analogue signal which can be transformed into a conditioning function by two discriminating threshold levels. The transitions between levels lead to the sequential enabling and disabling of the correlator. The strategy is illustrated schematically in Fig.1.

The thermocouple employed was constructed from a platinum/platinum-10\% rhodium wire combination, 50,m in diameter. On the scale of the flow disturbances studied, the necessary thermocouple positioning upstream of the LDA 
fringe volume in order to function as a trigger was not considered significant. By setting the temperature thresholds close together, with 50K separation, the digital correlator only accepts scattered light pulses from the LDA photomultiplier when the measured temperature lies within this narrowly defined range. Ensemble averaging of these conditioned events, namely local velocity $u$ given that temperature $T$ lies in the range $T_{C}-25 \leqslant T_{C} \leqslant T_{C}+25$ (K), permits a representation of the joint probability density function (pdf) for $u$ and $m$ to be constructed. This will be described more fully in the following section.

Between eight and ten temperature gates giving distinct velocities could typically be established, spanning the complete range of observed temperature fluctuations. The frequency response of the thermocouple was boosted by electrical compensation for the effects of thermal inertia in order to resolve local flame structure as clearly as possible.

Joint determinations of velocity and temperature from which turbulent correlations can be estimated are reported here in the form of radial traverses at two axial stations, $z=30 \mathrm{~cm}$. and $77 \mathrm{~cm}$. These locations distinguish approximately the lower and upper boundaries of the intermittently burning regime in the flame studied. Below $30 \mathrm{~cm}$. the buoyantly-accelerated flame column is comparatively wel1-defined whilst beyond $77 \mathrm{~cm}$. continuous flame is not observed and significant dilution and partial mixing occurs.

\section{Joint PDF Representation}

The narrowly defined temperature windows permit the representation of the temperature pdf as a series of delta functions, each centred within particular temperature thresholds. By measuring the relative proportions of temperature window open to closed (cf. Fig.1(c)) and scaling to the value of the measured mean temperature we construct the pdf. The velocity, associated with each conditioning temperature window and measured by the LDA, is determined in the form of a mean value and accompanying rms fluctuation. Given the strengths of the delta functions in the representation of the pdf for temperature, we can use these to weight the contributions of the conditional velocities to the overall velocity statistics. Comparisons between such estimates and unconditionaI velocity measurements at the same location permit alternative prescriptions for the joint pdf $P(u, T)$ to be tested. Two prescriptions are described in this paper; that the conditional velocity $\operatorname{pdf} P\left(u / T=T_{C}\right)$ is itself a delta function centred on the measured mean value or that $P\left(u / T=T_{0}\right)$ is Gaussian, having mean and variance as measured.

The joint paf $P(u, T)$ is given by

$P(u, T) \simeq P\left(u / T=T_{c}\right) P\left(T_{c}\right)$

where the discretised pdf for conditioning temperature is

$P\left(T_{c}\right)=\sum_{j=1}^{n} a_{j} \delta\left(T_{c}-T_{c}^{(j)}\right)$

and where $n$ denotes the number of temperature windows and $a_{j}$ is the strength of the delta function corresponding to window-centre temperature $T_{c}(j)$.

The conditional velocity distributions are then given either by

$P\left(u \mid T=T_{c}\right)=\delta(u-\vec{u}(j))$ or
$P\left(u \mid T=T_{c}\right)=\left\{\sqrt{\pi} \sigma^{(j)}\right\}^{-1} \exp \left\{-[u-\bar{u}(j)]^{2} / 2 \sigma^{(j)^{2}}\right\}$

where $\bar{u}^{(j)}, \sigma^{(j)^{2}}$ denote the measured mean and variance associated with 
conditioning temperature $T_{C}^{(j)}$.

Not unexpectedly, the Gaussian form leads to a marginal distribution for velocity on integration over $T_{C}$ which predicts more closely the measured velocity statistics in the absence of conditioning. The joint pdf $P\left(u, T^{\prime}\right)$ corresponding to this case is illustrated in Fig.2. It must be emphasised that since we are here triggering velocity data acquisition and ensemble averaging over events distinguished by temperature and not merely partitioning a single velocity time series, these comparisons are not trivial. The plausible reproduction of unconditioned statistics is however crucial if we are to have confidence in the representation of the joint pdf and hence in estimates of velocitytemperature correlations.

Figure 2 reveals a significant spread of velocities associated with each individual conditioning temperature and whilst there is a discernible displacement of conditional mean velocity, $\bar{u}(j)$, to higher values with increasing window temperature, there is also extensive overlapping. For conditioning temperatures in the range $298 \leqslant \mathbb{T}_{C} \leqslant 1887(\mathrm{~K}), 1.5 \leqslant \bar{u}^{(j)} \leqslant 5.6\left(\mathrm{~ms}^{-1}\right)$, such spread suggests that temperature alone would prove an unsuitable diagnostic for local conditions - an important factor in relation to the 'two-velocities' observed in the flame.

The LDA system detects the motion of naturally occurring particles, largely of soot, as they pass through the fringe field. Small particles, produced in the vicinity of the reaction zone, exhibit comparatively small axial velocities $\left(\vec{u}<2.6 \mathrm{~ms}^{-1}\right)$ and are subject to the more direct influence of the flapping flame. More aged, larger particles, though fewer in number, are more strongly accelerated in the buoyant fuel/product regions ( $\left(u<4.6 \mathrm{~ms}^{-1}\right.$ ). These two velocities can be distinguished at each location by varying the aperture of the collection optics - the larger the aperture the more significant the contribution from the large number of small particles accompanying the local flame zone and vice versa. Two distinct mean velocities are observed at most positions within the flame. The introduction of conditioning by local temperature as a further descriptor of local state, additional to particle number density and size, might be expected to offer some corroboration of this diagnosis. However, it proved impossible to overwhelm the seeding bias simply by displacing the temperature window and both velocities could be obtained over almost the complete range of conditioning temperature. Since temperature is not an unambiguous measure of local mixture state - the temperature mixture fraction relationship is double-valued, for example - this observation is not entirely unexpected, irrespective of any shortcomings in the measurement technique.

Table 1 presents comparisons at the $z=30 \mathrm{~cm}$. station between unconditional and conditional data using the alternative representations for the joint pdf described earlier. Whilst the temperature statistics are satisfactorily reproduced throughout, particularly in the centre of the flame, the velocities are less satisfactory. The marginal pdfs for velocity, cf. eqns. (3) and (4), constructed from conditional measurements, give mean velocities which compare reasonably well with unconditioned measurements - discrepancies $\lesssim 25 \%$ - but more significant differences emerge in relation to the rms values. The delta function representation leads to substantial under-estimates whilst the Gaussian form in general leads to over estimation. Given the difficulties generally encountered in velocity measurement in buoyant fires - the large amplitude, low frequency flapping motion inevitably poses problems in relation to reproducibility even in the absence of conditioning - the Gaussian joint pdf representation was deemed sufficiently good for useful estimates of turbulent correlations to be made.

The estimates of $\overline{u^{\prime} T^{\prime}}$ are obtained by quadrature, 


$$
\overline{u T}=\iint_{0}^{\infty} u T P\left(u \mid T=T_{c}\right) P\left(T_{c}\right) d u d T_{c}=\overline{u T T}+\overline{u T}
$$

The turbulent flux is positive at all the stations investigated, peaking off-axis at $z=30 \mathrm{~cm}$. but closer to the axis at $z=77 \mathrm{~cm}$. The mean temperature gradient is small, but positive, at $z=30 \mathrm{~cm}$. and then decreases such that $\frac{\partial \bar{T}}{\partial z}<0$ at $z=77 \mathrm{~cm}$. A simple gradient description

$\bar{\rho} \overline{u^{\prime} T^{\prime}}=-\frac{u_{t}}{\sigma_{T}} \frac{\partial \bar{T}}{\partial z}$

would evidently prove unsatisfactory at the lower station. Since the occurrence of buoyantly accelerated fluid elements may quite simply reflect flame motion and local heat release, any relationship to the mean temperature gradient is of limited mechanistic significance.

Comparisons with other measurements are restricted to non-combusting heated plumes. The relative flux intensity, $\overline{u^{\prime} T^{\prime}} /\left(\overline{u^{\prime 2}}\right)^{\frac{1}{2}}\left(\bar{T}^{\prime 2}\right)^{\frac{1}{2}}$, is comparatively insensitive to the particular application as shown in Fig. 3 and a broad measure of agreement is evident. At such levels the relative contribution of the turbulent heat flux, in comparison with the convective flux, might be expected to be small. The relative contributions are described in the following section.

\section{MASS AND HEAT FLUX BALANCES}

In estimating fluxes the contribution of the density is complex. Whilst the flame is substantially isobaric from the standpoint of thermodynamic state, in using methane as fuel we admit density variations resulting from both molecular weight and temperature changes. Beyond $z=30 \mathrm{~cm}$, the mean mixture fraction is everywhere sma11, $\bar{\xi}<0.1$ (7), and therefore the influence of molecular weight may be assumed small. We shall suppose $\rho=\rho(T)$ such that $\rho T=\rho_{0} T_{0}$, whence the mass flux crossing plane $z$ may be written

$$
\begin{aligned}
& \overline{\dot{m}}(z)=\int_{0}^{\infty} \overline{\rho u} d A=\int_{0}^{\infty}\left[\iint_{0}^{\infty} \rho(T) u \quad p(u, T) d T d u\right] d A \text {, and from eqns. (2) and (4) } \\
& \left.\overline{\rho u}=\sum_{j=1}^{n} a_{j} \rho^{\left(T T_{c}\right.}{ }^{(j)}\right) u^{(j)}=\sum_{j=1}^{n} a_{j} \rho_{0}\left\{\frac{T_{0}}{T_{e}^{(j)}}\right] \vec{u}^{(j)}
\end{aligned}
$$

Similar expressions apply to momentum and energy flux correlations

$\overline{\rho u^{2}}=\sum_{j=1}^{n} \overline{u^{(j)^{2}}} a_{j} \rho_{0}\left\{\frac{T_{0}}{T_{T}^{(j)}}\right\}$ and $\overline{\rho u T}=\sum_{j=1}^{n} a_{j} \overline{u^{(j)}} \rho_{0} T_{0}=\bar{u} \rho_{0} T_{0}$

In the absence of detailed information of the type reported here it has been. customary to neglect the turbulent contribution to such expressions. In such circumstances we would estimate the mass flux to be

$$
\dot{m}_{m}(z)=\int_{0}^{\infty} \bar{\rho} \bar{u} d A=\left\{\sum_{j=1}^{2} a_{j} \rho\left(T_{c}{ }^{(j)}\right)\right\} \sum_{j=1}^{m_{m}} a_{j} \bar{u}^{(j)}
$$

The turbulent contribution from eqns. (6) and (8) is then

$$
\overline{\rho^{\prime} u}=\overline{\rho u}-\bar{\rho} \bar{u}=\rho_{0} \sum_{0} \sum_{j=1}^{n} \frac{a_{j}}{T_{c}(j)}\left[\bar{u}^{(j)}-\sum_{j=1}^{n} a_{j} \bar{u}^{(j)}\right]
$$


The density-velocity correlation, estimated from eqn.(9), at two axial stations, $z=30$ and $77 \mathrm{~cm}$, is illustrated in Fig.4. The correlation is negative at both stations across the complete flame and typically $10 \%$ of the mean flux, $\bar{\rho} \bar{u}$. The results quoted relate specifically to the upper mean particle velocity but relative flux levels, $\bar{p}^{\prime} u^{\prime} / \rho \bar{u}$, are comparable for the lower velocity also. Despite the large-scale, buoyant flame motion the turbulent contribution is therefore indicated to be quite smal1. Whilst uncertainties surround the effect of molecular weight variations, not included in the density model, and the measurement of temperature fluctuations, these levels are believed to be broadly representative. The molecular weight effect might be incorporated using a flamelet profile and flapping flame model (cf. $(11,12)$ ) but in the present work we have elected to minimise the modelling element introduced directly into the data analysis.

Comprehensive mapping of the flame for velocity and temperature, including their joint determination described earlier, permits important features of zonal balances to be assessed. Fig.5 illustrates the data set in the form of radial profiles at axial stations $z=0,30$ and $77 \mathrm{~cm}$, the latter defining the lower and upper bounds of the intermittently burning regime. Additional data are reported elsewhere $(7,9)$.

Mass momentum and energy balances are investigated in terms of the properties summarised in Fig.6. The choice of a radius of $16 \mathrm{~cm}$. for the bounding cylinder is arbitrary. This particular value is broadly representative of the maximum flame width, attained in the neighbourhood of the $z=77 \mathrm{~cm}$. station, and permits the air drawn into the burning zone from beneath the burner to be featured in the 'source flow' at $z=0$.

The mass flux crossing the $z=0$ face, $\dot{m}(z=0)$, comprises $\dot{m}(z=0)=\dot{m}_{f}+\dot{m}_{a_{1}}=\pi R_{f}^{2} \rho_{f} u_{f}+2 \pi \int_{R=13}^{R=16} \rho_{a} u_{a 1} R d R$

since the turbulent contributions are neg1igible. Normalising with respect to the initial fuel flow, with $R^{*}=R / R$,

$\dot{m}(z=0) / \dot{m}_{f}=I+\left(\rho_{\alpha} / \rho_{f}\right) \int_{0}^{1} \cdot 5\left(u_{\alpha 1} / u_{f}\right) d R^{* 2}$

and substituting for the velocities (cf. Fig.5),

$\dot{m}_{(z=0)} \dot{m}_{f}=11.5$.

Given that stoichiometric methane combustion requires an air:fuel ratio of approximately 18:1 it might be argued that more than half the air required enters the flame through an annular region immediately surrounding the burner.However, the large amplitude flame flapping motion suggests that such literal interpretation of zonal balances of this type is potentially misleading. The mean radial inflow velocity, $\bar{v}$, in this region is shown in Fig.7. Whilst the fluctuating component is substantially larger than the mean, there are no significant temperature fluctuations and it appears plausible to neglect the velocity-density correlation. The mean flux into the reference cylinder over the height $0 \leqslant z \leqslant 30(\mathrm{~cm}), \dot{m}_{\alpha_{2}}$, is estimated from

$\dot{m}_{\alpha 2} \dot{m}_{f}=\left(\rho_{\alpha} / \rho_{f}\right) 2 R_{16}^{*} \int_{0}^{30} \bar{v}(z) d z / u_{f} R_{f} \simeq 36$.

The radial inflow induced by buoyancy is thus substantially greater than that drawn from below the burner and emphasises the importance of flame motion and fluctuating heat release on distributed burning. 
The fluxes across the intermittently burning stations, $z=30$ and $77 \mathrm{~cm}$, are made rather ambiguous by the observation that the velocity field is characterised by two distinctive velocities. However the density and area weighting favours the wings of the flame where the differences between the two velocities is smal1. The discrepancies between flux estimates is accordingly rather smaller than might have been expected. Given the comparative sparseness of the conditioned data, these fluxes are estimated from

$\dot{m}(z) / \dot{m}_{f}=\int^{1.5}\left\{\bar{\rho} \bar{u}+\overline{\rho^{\prime} u^{\prime}}\right\} d R^{* 2} / \rho_{f^{u}}$

with $\vec{\rho} \vec{u}$ determined from unconditioned measurements and $\bar{\rho}{ }^{\prime} u^{\prime}$ alone by conditional analysis (cf. Fig.4). We find

$\dot{m}(z=30) / \dot{m}_{f} \simeq 48$ (lower mean velocities) or $\simeq 50$ (upper mean velocities) values which compare favourably with the sum of the fluxes into the cylindrical. volume, bounded by the planes $z=0$ and $30 \mathrm{~cm}$, revealed in equations (10) and (11), namely 47.5 . we find

Adopting the same approach to the volume bounded by planes $z=30$ and $77 \mathrm{cms}$. $\dot{m}_{a_{3}} \dot{m}_{f} \simeq 73$ and $\dot{m}(z=77) / \dot{m}_{f} \cong 114$ (lower mean velocities) or 175 (upper mean

The difference between particle velocities is now significant across the complete radius and the flux estimates reflect this fact. Whilst the mean temperature is now falling (cf.Fig.5) and, increasingly, dilution mixing occurs, some burning is also observed. The flux across the plane $z=77 \mathrm{~cm}$, is at least five times that required for complete combustion, a factor again emphasising the largescale, intermittent nature of the flame.

Local apportioning of the scalar states giving rise to the two distinctive mean velocities has not proved possible. As indicated earlier the flux across $z=30 \mathrm{~cm}$. Is insensitive to the aperture setting although the lower mean velocity gives a slightly better mass balance. Scaling the upper/lower bound estimates crossing $z=77 \mathrm{~cm}$. to the net inflow suggests the relative contributions from flame zone and burnt gas to be in proportions 8:1. This ratio seems unexpectedly high, suggesting that the flame zone, lower bound velocity is the more representative of mass average behaviour even close to the top of the visible flame. It should be noted however that upper and lower mean velocities converge as $z$ increases (6). Also, the large radial in-flow, $\dot{m}_{\alpha 3}$, has been estimated from $\bar{p} \vec{v}$ in the absence of the radial velocity-density correlation. At $z=77 \mathrm{~cm}$. the turbulent contribution is less plausibly neglected than at $z=30 \mathrm{~cm}$. in view of

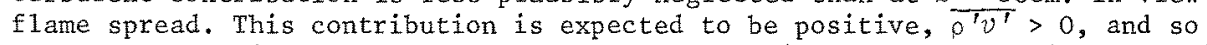
augment the radial inflow. Increased flux from this source will evidently shift the flame zone-to-burnt gas proportion towards the latter.

The momentum and energy balances are less complete. The mean sensible enthalpy flux, $\dot{q}(z)$, can evidently be calculated from conditioned measurements in a manner analogous to the mass fluxes reported earlier. For

$\dot{q}(z)=\int_{A} C p(\overline{\rho u T}) d A$,

assuming constant specific heat, $C p$, and the density model introduced earlier $\rho T=$ constant $=\rho_{0} T_{0}$ then 1.5

$\dot{q}(z) / \dot{Q}_{f}=\left(\rho_{0} / \rho_{f}\right)\left(Q / C p T_{0}\right) \int_{0}^{1}\left(\bar{u} / u_{f}\right) d R * 2$

where $\dot{Q}_{f}$ is the fuel source energy flow rate and $Q$ is the heat of reaction.

The normalised enthalpy fluxes crossing planes $z=30$ and $77 \mathrm{~cm}$. are found 
to be

$\dot{q}(z=30) / \dot{Q}_{f} \simeq 0.79$ (upper mean),$\simeq 0.59$ (1ower mean)
$\dot{q}(z=77) / \dot{Q}_{f} \simeq 1.95$ (upper mean),$\simeq 0.96$ (1ower mean)

Such estimates are dependent on the mean velocity profiles and the ambiguity introduced by upper and lower bounds is particularly marked at $z=77 \mathrm{~cm}$. Values substantially in excess of unity are clearly unphysical. We expect the zonal balances to be dominated by convective flux, chemical heat release rate $\left(\leqslant \dot{Q}_{f}\right)$ and radiative loss. The lower mean velocity again suggests the more plausible behaviour. Modelling assumptions such as constant mixture specific heat and molecular weight introduce additional uncertainties into these estimates. The zonal balances suggest that these uncertainties may be broadly comparable with the radiative loss. Markstein (13) suggests from observation of propane flames over a range of fuel flow rates that the radiative power is approximately 0.21 0.25 times the total heat release. The radiative loss from methane flames, appropriate to the present experiment, is typically smaller since the contribution from luminous radiation is substantially reduced. Bearing this in mind, the energy releases in the two zones $(0 \leqslant z \leqslant 30,30 \leqslant z \leqslant 77(\mathrm{~cm})$ ) appear to be roughly comparable.

The momentum balance contains several terms which are inaccessible to measurement, in particular those relating to pressure gradients and buoyancy. The quadratic nature of the momentum $f 1 u x, \overline{\rho u^{2}}$, results in estimates which are strongly influenced by differences in particle velocities. Normalised flux estimates from the lower mean velocities are

$\dot{p}(z) /\left\{A_{r \in \mathcal{f}} g \int_{z}^{z}\left\{\rho_{0}-\bar{\rho}_{\ell}\right\} d z^{\prime}=0.10(0 \leqslant z \leqslant 30 \mathrm{~cm}) ; \quad 0.26(30 \leqslant z \leqslant 77 \mathrm{~cm})\right.$

where Aref is the control volume cross sectional area and the centre-line mean density, $\rho_{\ell}$, is assumed to be given by

$$
\begin{aligned}
& \bar{\rho}_{Q}=\rho_{0}\left\{T_{0} \bar{T}_{q_{\max }}\right\} \quad(0 \leqslant z \leqslant 20(\mathrm{~cm})) \\
& =\rho_{0}\left\{T_{0} / T_{Q}\right\} \quad(z>20(\mathrm{~cm}))
\end{aligned}
$$

and $\rho_{0}$ is the ambient air density.

The use of centre-line density to model the buoyant contribution wi11 lead to an over-estimate of its effect since the mean density is a minimum there. Whilst the absolute values are evidently uncertain the relative contributions from the two zones are again broadly comparable. It was adjudged that a more detailed assessment of the buoyant contribution by integration of the radial profiles was unlikely to be more informative.

\section{CONCLUSTONS}

Gross inhomogenities in state and flow properties which are characteristic of buoyant flames make the turbulent flow field accessible to conditional sampling techniques. Temperatures measured by fine wire thermocouples are used to generate a strobe signal which triggers LDA data acquisition. Velocity statistics conditional on $10 \mathrm{cal}$ temperature result. These have been interpreted as a joint probability density function for velocity and temperature. Results of an extensive mapping of the flow field are reported.

The joint pdf is used to estimate the axial turbulent fluxes arising in mass, energy and momentum balances at two axial stations. The turbulent velocitytemperature correlation is revealed to be positive at both stations although the gradient of mean temperature changes sign. The accompanying mass flux $\rho^{\prime} u^{\prime}$ is 
negative but comparatively sma11, typically $10 \%$ of the flux estimated from mean properties, $\vec{\rho} \vec{u}$.

Results of a simple control volume analysis for cylindrical volumes bounded by planes $z=30$ and $77 \mathrm{~cm}$. are presented. Some uncertainty is introduced into the analysis by the observation of two mean particle velocities. The smaller of these corresponds to the high number density of smal1 particles in the vicinity of the burning zone whilst the higher velocity is associated with the lower density of larger, aged particles characteristic of fuel-product regions. Two zonal balances suggest that the lower of these velocities is the more representative in the region where the two velocities differ significantly.

The radial inflow, induced by large-scale flame motion and buoyancy, is shown to be the principal component in the mass balances. However, a significant air flow is induced from beneath the burner through an annular region immediately surrounding it. The mass flux through the top of the control volume at $z=77 \mathrm{~cm}$. is at least five times that required for complete combustion, illustrating the large scale intermittent structure of the flame. The energy and momentum balances are incomplete but the flux estimates suggest that chemical heat release and buoyant acceleration are broadly comparable in the two zones. The radiative loss cannot be distinguished from the uncertainties introduced in modelling, notably the velocity ambiguity and molecular weight effects on the density field.

\section{REFERENCES}

1. Becker, H.A. and Yamazaki, S. (1978). Entrainment, Momentum Flux and Temperature in Vertical Free Turbulent Diffusion Flames. Comb. and Flame 33, 123.

2. McCaffrey, B.J. (1979). Purely Buoyant Diffusion Flames: Some Experimental Results. National Bureau of Standards, NBSIR 79-1910.

3. Cox, G. and Chitty, R. (1980). A Study of Deterministic Properties of Unbounded Fire Plumes. Comb and Flame 39, 191.

4. You and Faeth,G.M. (1982). Buoyant Axisymmetric Turbulent Diffusion Flames in Sti11 Air. Comb. and Flame 44, 261.

5. Gengembre, E., Cambray, P., Karmed, D. and Be1let, J-C. (1983). Turbulent Diffusion Flames with Large Buoyancy Effects. Paper presented at IXth ICODERS, Poitiers.

6. Walker, N.L. and Moss, J.B. (1984). LDA Measurement in and around a Turbulent Buoyant Flame. Comb. Sci. and Tech. 17, 65.

7. Crauford, N.L., Liew, S.K. and Moss, J.B. (1985). Simulated Buoyant Fire Modelling and Experiment. Comb. and Flame. (to appear).

8. Nakagome, H. and Hirata, M. (1976). The Structure of Turbulent Diffusion in an Axi-symmetrical Thermal plume. Heat Transfer and Turbulent Buoyant Convection (eds. Spalding, D.B. and Afgan, S.)

9. Crauford, N.L. (1984). The Structure of an Unconfined Buoyant Turbulent Diffusion Flame. Ph.D. Thesis, Southampton University.

10. Ballantyne, A. and Moss, J.B. (1977). Fine Wire Themocouple Measurements of Fluctuating Temperature. Comb. Sci. and Tech. 17, 65.

11. Roberts, P.T. and Moss, J.B. (1981). A Wrinkled Flame Interpretation of the Open Turbulent Diffusion Flame. Eighteenth Symposium (International) on Combustion, p.941, Combustion Institute.

12. Liew, S.K., Bray, K.N.C. and Moss, J.B. (1981). A Flamelet Model of Turbulent Non-premixed Combustion, Comb. Sci. and Tech. 27, 69.

13. Markstein, G.H. (1977). Scaling of Radiative Characteristics of Turbulent Diffusion Flames. Sixteenth Symposium (International) on Combustion, 
p, 1407, Combustion Institute.

14. George, W.K., Albert, R.L. and Tamanini, F. (1977). Turbulence Measurements in an Axisymmetric Buoyant P1ume. Int.Journal Heat and Mass Transfer 20, 1145.

\section{ACKNOWLEDGEMENTS}

This paper forms part of the work of the Fire Research Station, Building Research Establishment, Department of the Environment, U.K. It is contributed by permission of the Director, Building Research Establishment. Helpful discussions with Mr.G.Cox, Fire Research Station are gratefully acknowledged. Crown Copyright 1985.

\begin{tabular}{|c|c|c|c|c|c|c|c|c|}
\hline \multirow{2}{*}{$\frac{\text { Position, } \mathrm{R}(\mathrm{cm})}{\bar{u}\left(\mathrm{~ms}^{-1}\right)}$} & \multicolumn{2}{|c|}{$\begin{aligned} & p=0 \\
& \text { Lower/upper velocities }\end{aligned}$} & \multicolumn{2}{|c|}{$\begin{array}{c}\mathrm{g}=4 \\
\text { Lower/upper }\end{array}$} & \multicolumn{2}{|c|}{ 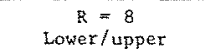 } & \multicolumn{2}{|c|}{$\begin{array}{c}\mathrm{R}=12 \\
\text { Lower/upper }\end{array}$} \\
\hline & 2.27 & 3.94 & 1.73 & 2.75 & 0.71 & 0.74 & 0.32 & 0.33 \\
\hline $\bar{u}_{\theta}\left(=\bar{u}_{G}\right)$ & 2.69 & 3.59 & 2.10 & 2.22 & 1.07 & 0.98 & 0.30 & 0.32 \\
\hline$(u-2)^{k}$ & 0.75 & 0.93 & 0.55 & 0.96 & 0.35 & 0.37 & 0.24 & 0.24 \\
\hline$\left(\overline{u^{-2}}\right)_{\delta}^{\frac{3}{2}}$ & 0.29 & 0.49 & 0.27 & 0.41 & 0.34 & 0.32 & 0.12 & 0.12 \\
\hline$\overline{\left(\hat{u}^{-2}\right)_{G}^{\frac{1}{2}}}$ & 1.11 & 1.11 & 0.80 & 0.80 & 0.60 & 0.60 & 0.26 & 0.26 \\
\hline $\bar{T}(\mathrm{~K})$ & \multicolumn{2}{|c|}{1225} & \multicolumn{2}{|c|}{946} & \multicolumn{2}{|c|}{757} & \multicolumn{2}{|c|}{453} \\
\hline $\bar{m}_{\delta}$ & \multicolumn{2}{|c|}{1221} & \multicolumn{2}{|c|}{943} & \multicolumn{2}{|c|}{656} & \multicolumn{2}{|c|}{401} \\
\hline$\left(\overline{(p-2)^{\frac{2}{2}}}\right.$ & \multicolumn{2}{|c|}{304} & \multicolumn{2}{|c|}{358} & \multicolumn{2}{|c|}{311} & \multicolumn{2}{|c|}{224} \\
\hline$\overline{(T-2)})_{\delta}^{3}$ & \multicolumn{2}{|c|}{325} & \multicolumn{2}{|c|}{353} & \multicolumn{2}{|c|}{304} & \multicolumn{2}{|c|}{1.58} \\
\hline $\bar{u} T^{T}$ & $93.5 \mathrm{I}$ & 114.61 & 88.70 & 114.54 & 92.68 & 84.77 & 20.90 & 20.18 \\
\hline$\overline{u^{\prime} T^{3}} / u_{\delta}{ }_{\delta}$ & 0.028 & 0.026 & 0.045 & 0.055 & 0.128 & 0.128 & 0.174 & 0.157 \\
\hline
\end{tabular}

$\delta, G$ subscripte denote estimates from conditional data using delta function and Gaussian representations for velocity, cf. eqs. (3) and (4), respectively.

TABIE 1. Velocity and Temperature from Conditioned data at $z=30 \mathrm{~cm}$.

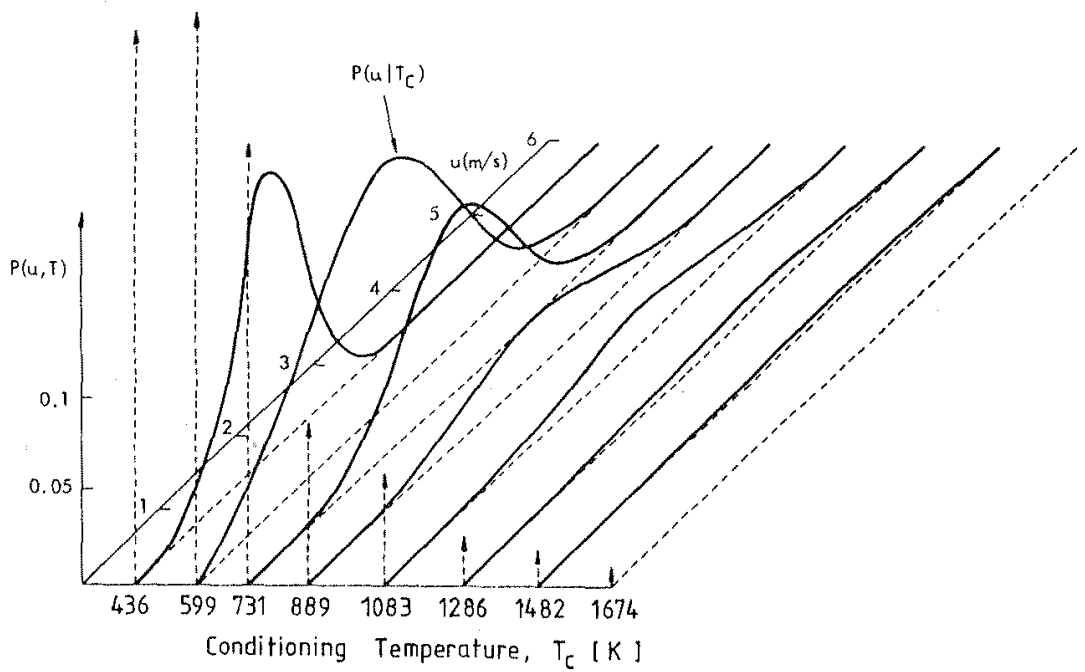

FIGURE 2. Joint Pdf for conditionally sampled velocity at $z=77 \mathrm{~cm}, R=0$, upper mean velocity. 

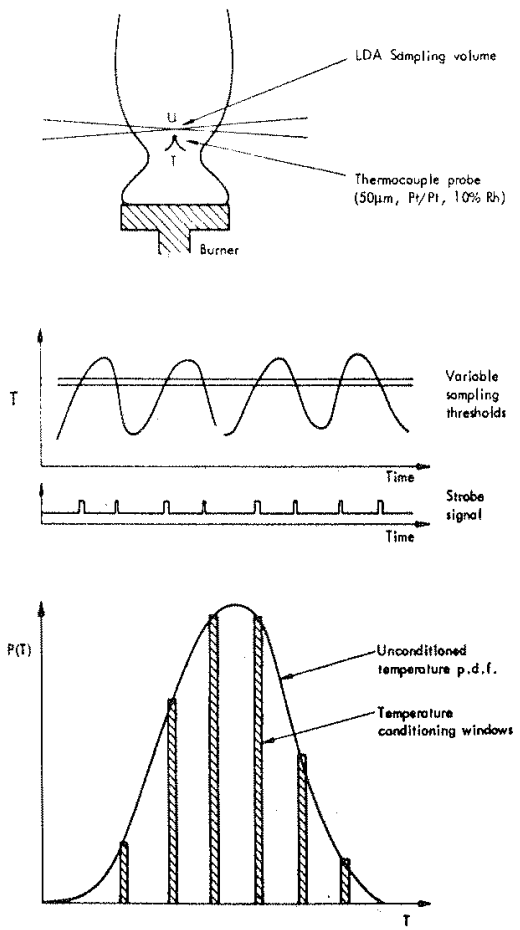

FIGURE 1. Schematic of conditional sampling strategy.

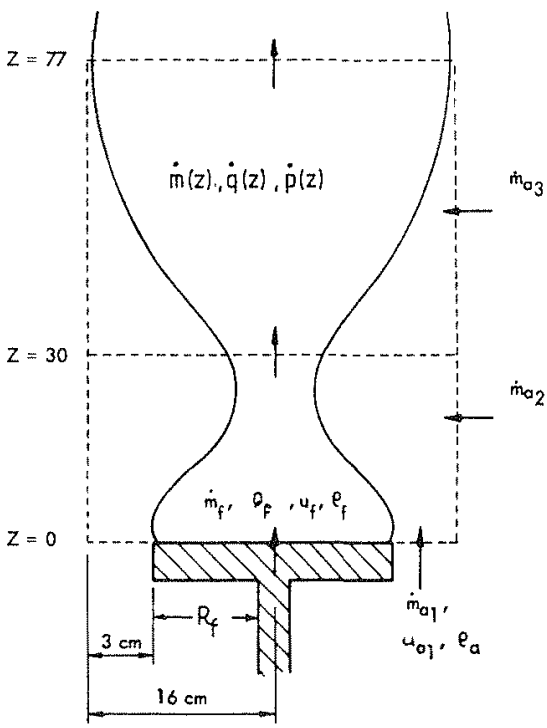

FIGURE 6. Nomenclature for control volume analysis.

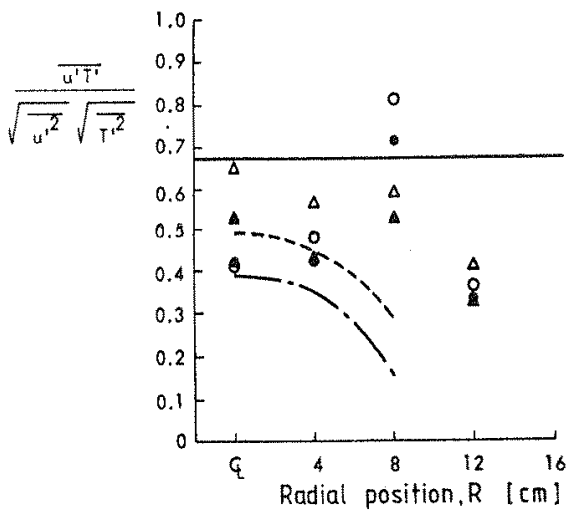

FIGURE 3. Heat flux intensity comparisons. Present experiments

$z=30 \mathrm{~cm}, 0$, upper $/$ lower mean velocities $z=77 \mathrm{~cm}, \Delta, \Delta$
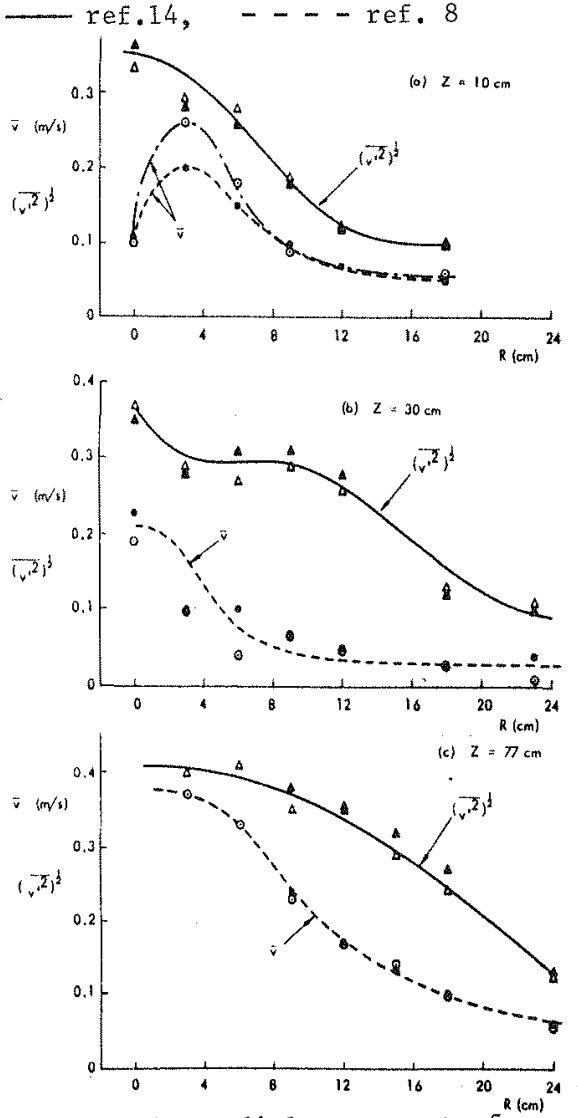

FIGURE 7. Radial component of mean and rms velocity. 

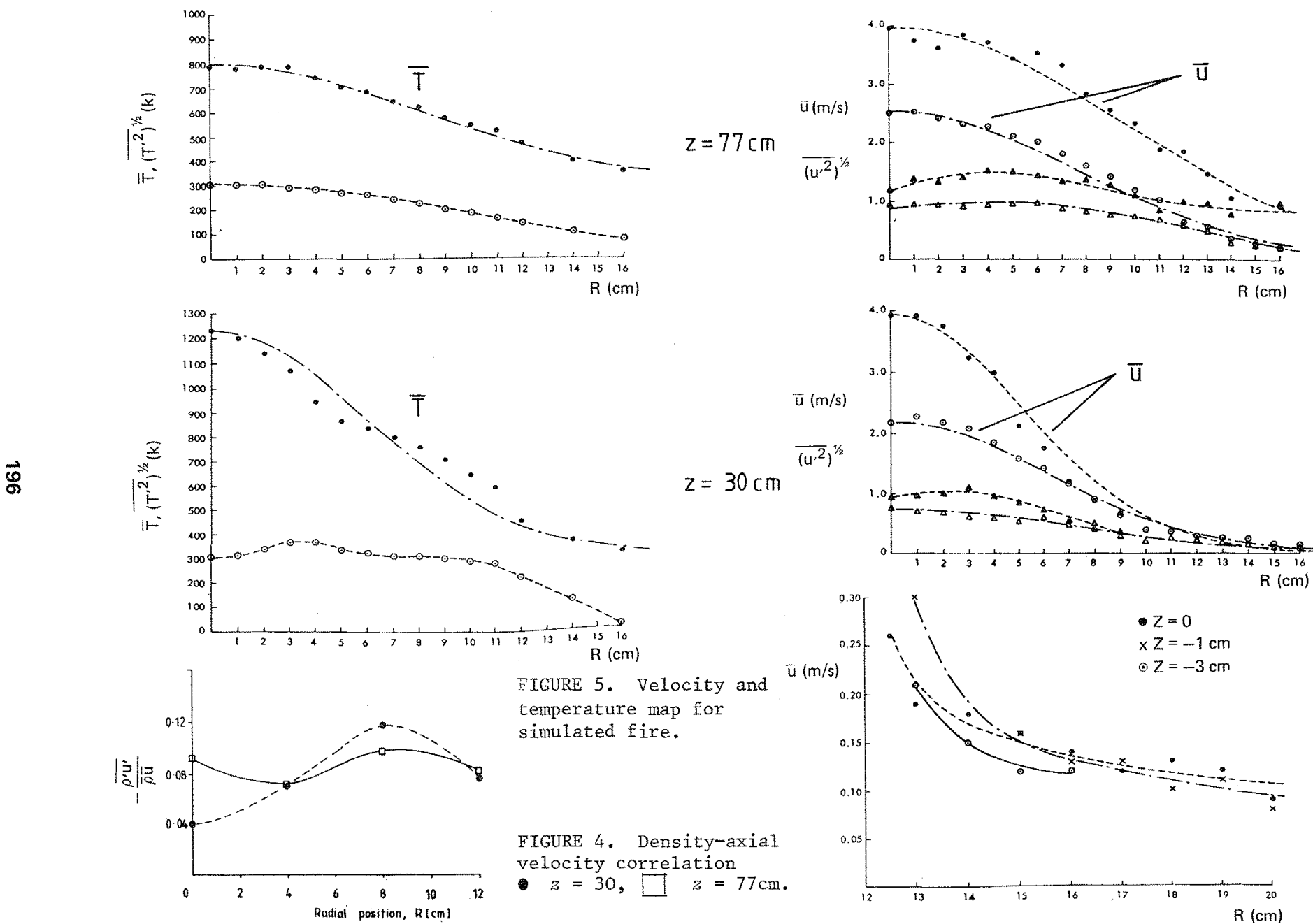